\title{
Billing, Coding and Credentialing for Interventional Radiology-Operated Endoscopy
}

Jesse Mer, MD, MBA ${ }^{1}$; Jeffrey Forris Beecham Chick, MD, MPH, DABR ${ }^{2}$; Travis L. Healey, MD ${ }^{1}$; Evan J. Johnson, MD $^{3}$; Ravi N. Srinivasa, MD, FSIR ${ }^{4}$

${ }^{1}$ Department of Radiology and Imaging Sciences, Indiana University School of Medicine

${ }^{2}$ Division of Vascular and Interventional Radiology, Inova Alexandria Hospital, 4320 Seminary Road, Alexandria, VA 22304

${ }^{3}$ Department of Radiology, Mayo Clinic, 200 First St. SW, Rochester, MN 55905

${ }^{4}$ Department of Radiology, Division of Vascular and Interventional Radiology, UCLA Medical Center, 757 Westwood Plaza, Los Angeles, CA 90095

This is the author's manuscript of the article published in final edited form as:

Mer, J., Chick, J. F. B., Healey, T. L., Johnson, E. J., \& Srinivasa, R. N. (2019). Billing, Coding, and Credentialing for Interventional Radiology-Operated Endoscopy. Techniques in Vascular and Interventional Radiology. https://doi.org/10.1053/j.tvir.2019.04.009 
Abstract

A sound understanding of billing and coding is essential to starting a successful interventional radiology endoscopy practice. While the codes utilized are similar to gastrointestinal and genitourinary endoscopy codes, physicians and institutional coders need to be familiar with the codes used for these types of procedures in the interventional radiology setting. The following manuscript gives a brief overview of aspects relating to credentialing, billing, and coding in interventional radiology endoscopy.

KEYWORDS: Billing; coding; interventional radiology; endoscopy; laser

ABBREVIATIONS: IR - interventional radiology; CPT - current procedural terminology; SIR - Society of Interventional Radiology; ACR - American College of Radiology; ASLMS - American Society for Laser Medicine and Surgery 
The diagnostic and therapeutic value of endoscopic methods in the interventional suite is becoming realized in a number of radiology departments across the country. Yet the organizational infrastructure required for widespread employment of these methods in the US is not complete. From a billing standpoint, there is already a coding framework for many interventional radiology (IR) procedures using endoscopy. Training pathways for radiologists to use endoscopy are still however not fully defined and there is opportunity to learn from models abroad. Here we outline some of the challenges and strategies for broadening the adoption of endoscopic methods in the interventional suite. Specifically, we will briefly discuss laser and endoscopy credentialing for the interventional radiologist as well as some specific CPT codes for use.

One of the most significant obstacles for widespread adoption of endoscopy in interventional radiology is the current lack of training opportunity. There are several workshops across the country dedicated to endoscopic training for the radiologist; however, a broader solution is necessary - one capable of guiding physicians on a national level. There are models that can be taken from abroad, such as those developed by the British Society of Gastrointestinal and Abdominal Radiology: Britain's National Health Service provides pathways for the radiologist to certify in upper gastrointestinal endoscopy. For political reasons, equivalent opportunities for US radiologists may not be realistic, but the British example does highlight the benefit of organizational support. Potential solutions for the incorporation of endoscopy training for US radiologists may involve our own professional organizations such as Society of Interventional Radiology (SIR) or the American College of Radiology (ACR). For those radiologists who are already using endoscopic techniques, maximizing teaching opportunities for residents and colleagues alike is another method by which this technology can be introduced into the field.

For laser therapies, there are well-established methods for credentialing in the US as governed by The American Society for Laser Medicine and Surgery (ASLMS). A minimum of 8 to 10 hours of basic training is recommended, at least $40 \%$ of which should be based on practical sessions. Instruction should cover laser physics, tissue interaction, safety, surgical techniques, and pre-and postoperative care. These structured courses should be complemented by preceptorship-type clinical training. Alternatively, completion of a residency program that has included ample training and practice in laser techniques should be considered adequate for laser privileges. Granting and maintaining privileges should remain the responsibility of the hospital or institution, with protocols and procedures in compliance with the laws of each state as well as ASLMS guidelines. 
Consulting SIR's latest Interventional Radiology Coding Update is the best place to start for coding. This document is compiled by the Society of Interventional Radiology for free use by its members. It is continually updated to reflect the widening array of services provided by the field. The SIR 2019 Coding Update outlines multiple dedicated codes relating to percutaneous biliary endoscopy (Table 1). For renal endoscopy, much of the coding can be borrowed from urology (Table 2).

The augmentation of current interventional radiology techniques with endoscopy can be of great benefit to many patients. The most significant barrier to the broader adoption of endoscopy in the IR suite relates to the limited training available in the US. Deliberate efforts by radiology's professional organizations may be a strategy to overcoming this obstacle. Additionally, for those select institutions already employing these methods, all opportunities should be taken to instruct colleagues and trainees. With such efforts across the field, percutaneous endoscopy can become a more commonplace technique in certain challenging cases, furthering the capability of the interventional radiologist to provide the most innovative and quality patient care. 


\section{REFERENCES:}

1. American Society for Laser Medicine and Surgery (ASLMS).

http://www.aslms.org/for-professionals/professional-resources/standards-ofpractice/standards-of-training-for-physicians-for-the-use-of-lasers-in-medicineand-surgery

2. SIR - Practice Resources - Coding Update 2019. https://www.sirweb.org/practice-resources/

3. Chick JFB, Cooper K, Srinivasa RN. Interventional Radiology-Operated Endoscopy as an Adjunct to Image-Guided Interventions. Curr Probl Diagn Radiol. 2018 Mar 20. pii: S0363-0188(18)30048-3. doi: 10.1067/j.cpradiol.2018.03.002. [Epub ahead of print] Review. PMID: 29674012

4. American Medical Association. (2019). AMA Cúrrent Procedural Terminology Professional Edition. AMA. 
Table 1 - Biliary endoscopy codes ${ }^{4}$

\section{CPT Code CPT Description}

Biliary endoscopy, intraoperative (choledochoscopy). (+List separately in

(+) 47550 addition to code for primary procedure).

Biliary endoscopy, percutaneous via T-tube or other tract; diagnostic, with collection of specimen(s) by brushing and/or washing, when performed 47552 (separate procedure)

Biliary endoscopy, percutaneous via T-tube or other tract; with biopsy, 47553 single or multiple

Biliary endoscopy, percutaneous via T-tube or other tract; with removal of 47554 calculus/calculi

Biliary endoscopy, percutaneous via T-tube or other tract; with dilation of 47555 biliary duct stricture(s) without stent

Biliary endoscopy, percutaneous via T-tube or other tract; with dilation of biliary duct stricture(s) with stent. (+ 47801 for placement of choledocal 47556 stent)

If imaging guidance is performed, use 74363

For endoscopic brushings, see 43260, 47552.

For endoscopic biopsy, see 43261, 47553.

For endoscopic removal of calculi, see 43264, 47554.

For endoscopic destruction of calculi, use 43265. 
Table 2 - Renal endoscopy codes $^{4}$

CPT Code CPT Description

Renal endoscopy through established nephrostomy or pyelostomy, w/ or w/o irrigation, instillation, or ureteropyelography, exclusive of radiologic

50551 service;

Renal endoscopy through established nephrostomy or pyelostomy, w/ or w/o irrigation, instillation, or ureteropyelography, exclusive of radiologic

50553 service; with ureteral catheterization, w/ or w/o dilation or ureter

For image-guided dilation of ureter and/or renal pelvis without endoscopic guidance, use 50606.

Renal endoscopy through established nephrostomy or pyelostomy, w/ or w/o irrigation, instillation, or ureteropyelography, exclusive of radiologic

50555 service; with biopsy

Renal endoscopy through established nephrostomy or pyelostomy, w/ or w/o irrigation, instillation, or ureteropyelography, exclusiye of radiologic

50557 service; with fulgration and/or incision, w/ or w/o biopsy

Renal endoscopy through established nephrostomy or pyelostomy, w/ or w/o irrigation, instillation, or ureteropyelography, exclusive of radiologic

50561 service; with removal of foreign body or calculus

Renal endoscopy through established nephrostomy or pyelostomy, w/ or w/o irrigation, instillation, or ureteropyelography, exclusive of radiologic

50562 service; with resection of tumor

Renal endoscopy through nephrotomy or pyelotomy, w/ or w/o irrigation, 50570 instillation, or ureteropyelography, exclusive of radiologic service;

Renal endoscopy through nephrotomy or pyelotomy, w/ or w/o irrigation, instillation, or ureteropyelography, exclusive of radiologic service; with

50572 ureteral catheterization, w/ or w/o dilation of ureter

Renal endoscopy through nephrotomy or pyelotomy, w/ or w/o irrigation, instillation, or ureteropyelography, exclusive of radiologic service; with

50574 biopsy

Renal endoscopy through nephrotomy or pyelotomy, w/ or w/o irrigation, instillation, or ureteropyelography, exclusive of radiologic service; with endopyelotomy (includes cystoscopy, ureteroscopy, dilation of ureter and ureteral pelvic junction, incision of ureteral pelvic junction and insertion of

50575 endopyelotomy stent)

Renal endoscopy through nephrotomy or pyelotomy, w/ or w/o irrigation, instillation, or ureteropyelography, exclusive of radiologic service; with

50576 fulgration and/or incision, w/ or w/o biopsy

Renal endoscopy through nephrotomy or pyelotomy, w/ or w/o irrigation, instillation, or ureteropyelography, exclusive of radiologic service; with

50580 removal of foreign body or calculus 